\title{
Laparoscopic completely extraperitoneal repair of inguinal hernia in children: a single-institute experience with 1,257 repairs compared with cut-down herniorrhaphy
}

\author{
Masao Endo • Toshihiko Watanabe • \\ Miwako Nakano • Fumiko Yoshida • \\ Etsuji Ukiyama
}

Received: 23 February 2008/ Accepted: 24 July 2008/Published online: 3 April 2009

(c) The Author(s) 2009. This article is published with open access at Springerlink.com

\begin{abstract}
Background Conventional open herniorrhaphy in children has been reported to have $0.3-3.8 \%$ recurrence and 5.6-30\% postoperative contralateral hernia rates. We developed a unique technique to achieve completely extraperitoneal ligation of PPV without any skip areas under laparoscopic control. This report introduces our technique and results compared with the cut-down herniorrhaphy.

Methods A consecutive series of 1,585 children with inguinal hernia/hydrocele (1996-2006) was analyzed. In laparoscopic patent processus vaginalis (PPV) closure (LPC), an orifice of PPV was encircled with a 2-0 suture extraperitoneally by a specially devised Endoneedle and tied up from outside of the body achieving completely extraperitoneal ligation of the ring. The round ligament was included in the ligation, whereas the spermatic cord and testicular vessels were excluded by advancing the needle across them behind the peritoneum. Cut-down herniorrhaphy (CD), with or without diagnostic laparoscopy, or LPC was selected according to parental preference under informed consent.

Results Parents gave more preference to LPC (LPC in 1,257 children, $\mathrm{CD}$ in 308 , and miscellaneous in 20). Age ranges were equal for both groups. Sex distribution showed female preponderance in the LPC group $(44.8 \%$ vs. $26.6 \%$, $p<0.001)$ and umbilical hernia/cysts were predominantly
\end{abstract}

\footnotetext{
M. Endo $(\bowtie) \cdot$ T. Watanabe $\cdot$ M. Nakano

Department of Pediatric Surgery, Saitama City Hospital,

2460, Mimuro, Midori-ku, Saitama-shi 336-8522, Japan

e-mail: emta@mxr.mesh.ne.jp

F. Yoshida $\cdot$ E. Ukiyama

Department of Pediatric Surgery, Kyorin University Hospital,

Mitaka-shi, Tokyo, Japan
}

included in the LPC group $(11.9 \%$ vs. $2.9 \%, p<0.001)$. Mean operation times were equal for both groups for unilateral repair $(28.2 \pm 9.2$ for LPC vs. $27.8 \pm 13.5$ for $C D)$ and were shorter for bilateral repair in the LPC group $(35.8 \pm 11.6$ vs. $46.7 \pm 17.7)$. The incidence of postoperative hernia recurrence and contralateral hernia in the LPC group was $0.2 \%$ and $0.8 \%$. Two children in the CD group had injuries to their reproductive system during the operation $(0.6 \%)$.

Conclusions The advantages of our technique include following: technically simple, short operation time, inspection of bilateral IIRs with simultaneous closure of cPPV, reproductive systems remain intact, routine addition of umbilicoplasty if desired, and essentially indiscernible wounds.

Keywords Laparoscopic herniorrhaphy . Indirect inguinal hernia - Extraperitoneal repair . Cut-down herniorrhaphy $\cdot$ Laparoscopic repair

The principle for the repair of indirect inguinal hernias in children consists of complete ligation of the patent processus vaginalis (PPV) [1]. For a long time, procedures with this goal had been unchangingly performed through an inguinal crease incision as the traditional cut-down technique [2]. With the advent of the laparoscopic era, the trend began to move toward the application of laparoscopic techniques for pediatric herniorrhaphy. Considerable debates currently exist about whether the laparoscopic approach to the indirect inguinal hernia should be allowed to take the place of the "gold standard."

Conventional open herniorrhaphy in children has been reported to have recurrence rates of $0.8-3.8 \%$ and postoperative contralateral hernia rates of $5.6-30 \%$ [3-6]. We 
developed a unique technique to achieve completely extraperitoneal ligation of the PPV without any skip areas, sparing the spermatic cord and testicular vessels under laparoscopic control. Our technique should offer theoretically superior advantages to open repair in terms of high ligation of the PPV and routine intervention to the contralateral side. The purpose of this report is to introduce our technique and results compared with conventional cutdown herniorrhaphy.

\section{Materials and methods}

Study design

A consecutive series of 1,585 children with inguinal hernia or hydrocele, or both, experienced during 1996-2006, was analyzed. Regarding the operative procedures, the cutdown procedure (CD) and laparoscopic PPV closure (LPC) were proposed to the parents of the patients. CD was further divided into $\mathrm{CD}$ for the affected side only (CDA) and $\mathrm{CD}$ with diagnostic laparoscopy (CDL). CDA, CDL, or LPC was selected according to parental preference under informed consent. The medical records of these children were analyzed in terms of parental selection, distribution of sex, age, presence of contralateral patent processus vaginalis (cPPV), operation time, and complications among the groups.

Twenty patients who had various procedures during the period of development of laparoscopic herniorrhaphy were excluded from the analysis, and patients who underwent combined procedures affecting definitive herniorrhaphy also were excluded from the analysis of the operation time. The patients were followed up regularly in our outpatient clinic until 7 months, and at the visit for any complaints or other morbidities after that time. The follow-up periods ranged from 1 to 11 years.

\section{Statistical analysis}

Continuous data were expressed as mean \pm standard deviation (SD). Statistical significance was calculated with a two-tailed $t$ test or the Mann-Whitney $U$ test. For proportion data, the $\chi^{2}$ test was used.

Operative procedures

In laparoscopic PPV closure, a 2-0 suture, placed in the lower half of the internal inguinal ring through a 16-gauge sheath needle advanced extraperitoneally across the cord and vessels, was retrieved through the upper half of the ring by a specially devised needle (Fig. 1) and was tied up extracorporeally, achieving completely extraperitoneal

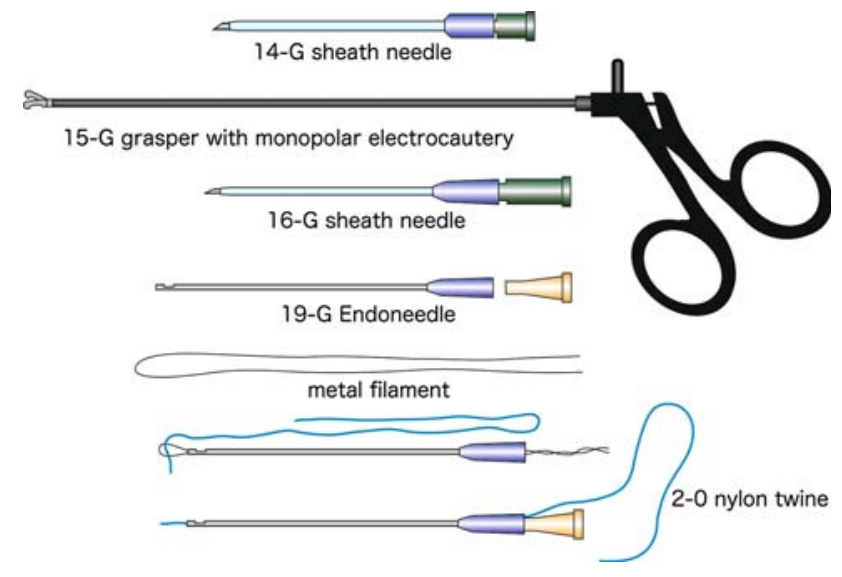

Fig. 1 Instrumentation consisting of 14-gauge sheath needle as a port for 15-gauge grasper with electrocautery, 16-gauge sheath needle for puncture, and 19-gauge Endoneedle for sending and retrieving a suture. A metal filament is used for setting a 2-0 nylon twine as a suture into the Endoneedle

ligation of the ring. Technical details are illustrated in Fig. 2. The procedure for girls was as described previously [7]. For infants younger than aged 1 year 6 months, the IIR was closed with double ligation (Fig. 3). If a cPPV was identified, it was closed.

In cut-down herniorrhaphy, the traditional procedure described in the text was performed through a crease incision at the affected side [8]. Diagnostic laparoscopy was performed with a 70-degree laparoscope via ipsilateral hernia sac. A 2-mm grasper was inserted through the paraumbilical port to manipulate the peritoneum at the contralateral IIR if needed. When a cPPV was noted, it was closed through a crease incision.

\section{Results}

Parental perspective and choice

Parents showed a greater preference for LPC, accounting for 1,257 children compared with 308 who underwent CD with or without diagnostic laparoscopy (CDA, 62; CDL, 246). The reasons why the parents chose LPC were postoperative cosmetic superiority, inspection for cPPV, and simultaneous repair if it was present, the ability for a second look at the previous operation site in cases of recurrence or contralateral occurrence, availability of simultaneous umbilicoplasty for umbilical hernia, or an ugly umbilicus. Diagnostic laparoscopy was selected for inspection for cPPV and simultaneous repair if it was present. The cut-down procedure was chosen because of strong disagreement of family members, including grandparents and relations based on fear of laparoscopic procedures, experience regarding siblings or other family 


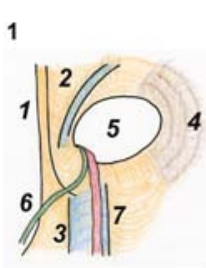

3

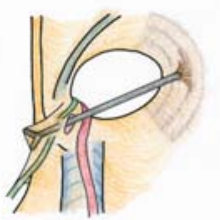

5

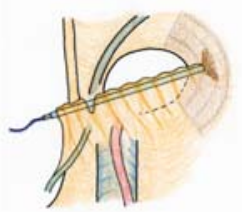

2
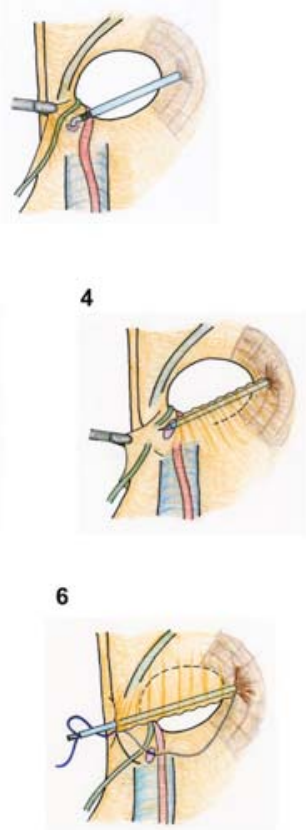

7

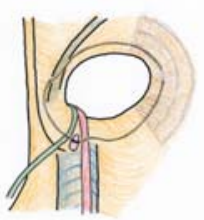

8

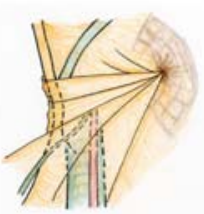

Fig. 2 Laparoscopic completely extraperitoneal closure of rightsided PPV. (1) Anatomy of male IIR. 1, umbilical plica; 2, inferior epigastric vessels; 3 , external iliac vein; 4 , transverse abdominal muscle; 5, orifice of PPV; 6, spermatic duct; 7, testicular vessels. (2) A small opening is made on the peritoneum between spermatic duct and testicular vessels using 15-gauge grasper with electrocautery. (3) The spermatic duct is separated from covering peritoneum by the grasper. (4) 16-gauge sheath needle goes along lower half of the IIR extraperitoneally crossing over the testicular vessels and spermatic duct beneath the peritoneum. (5) After the puncture needle penetrates the peritoneum at the opposite side, a $2-0$ suture is send by Endoneedle. (6) Free end of the suture is bitten into the Endoneedle that has come along upper half of the orifice and drawn out together with the needle. (7) The orifice of PPV has been encircled without any skip areas. The suture is tied from outside. (8) End of the procedure

members who had previously undergone cut-down herniorrhaphy with good results or no problems, and the lack of long-term follow-up data with the innovative technique.

Characteristics of patients who underwent each chosen procedure

The data are shown in Table 1. Age ranges were equal at approximately 3 years for both the LPC and CD groups. Sex distribution showed female predominance in the LPC group (44.8\% in LPC vs. $26.6 \%$ in CD, $p<0.001$ ).

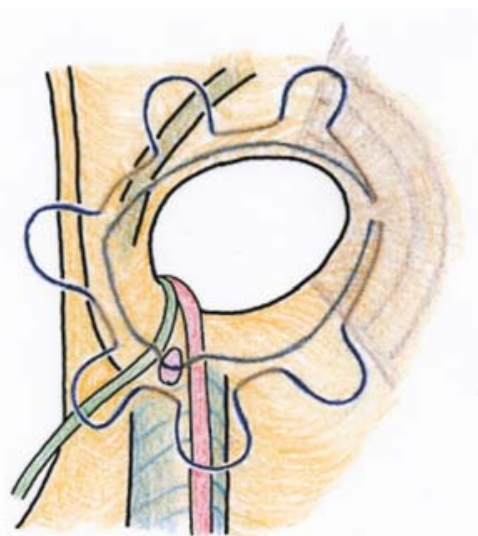

Fig. 3 Double ligation for infant younger than aged 1 year 6 months. An internal pursestring suture is placed, skipping over the spermatic cord and testicular vessels, proximally to the previously placed encircling suture

Differences in the hernia side were not significant between the groups. No statistically different distributions of associated morbidities necessitating combined operation were seen, except umbilical hernia/cyst. Umbilical morbidities, such as umbilical hernia, cystic degeneration of the umbilicus, or an ugly-looking umbilicus, were seen four times more in the LPC group $(p<0.001)$.

Operative findings

Regarding laparoscopic inspection of the contralateral IIR, the presence of cPPVs was more dominant in the LPC group than in the CDL group $(47 \%$ vs. $21.6 \%, p<0.001)$. Mean operation times for unilateral repair were equal in both LPC and CD groups $(28.2 \pm 9.2 \mathrm{~min}$ for LPC vs. $27.8 \pm 13.5 \mathrm{~min}$ for $\mathrm{CD}$ ) and were shorter for LPC in bilateral repair $(35.8 \pm 11.6$ vs. $46.7 \pm 17.7 \mathrm{~min}$, $p<0.001)$. When comparing the CDL with the CDA, CDL took an average of 6 min longer for a unilateral and 8.6 min longer for a bilateral closure than CDA $(p<0.05)$. The difference between males and females was significant in the LPC group $(p<0.001)$, accounting for a $4.4-\mathrm{min}$ increase for unilateral and a 6.9-min increase for bilateral closure in males (Table 2). In the LPC group, incidental umbilical hernia, ugly umbilicus, and other abnormalities, such as intraumbilical epidermoid cysts, also were repaired simultaneously during closure of the laparoscopic wound, whereas in the $\mathrm{CD}$ group an umbilicoplasty was performed as another definitive surgery.

One boy in the LPC group had a small stab injury on the anterior wall of the rectum during the placement of a port for the grasper through the abdominal wall, which was immediately repaired with the laparoscopic technique without sequelae. A boy and a girl in the CD group had respective accidental severance of the 
Table 1 Characteristics of patients who underwent chosen procedure

\begin{tabular}{|c|c|c|c|}
\hline & LPC & $\mathrm{CD}$ & Difference \\
\hline No. of patients & 1,257 & 308 (246 CDL, $62 \mathrm{CDA})$ & \\
\hline Age (range) & 1 month to 24 years & 1 month to 22 years & \\
\hline Mean $\pm \mathrm{SD}$ & $3.8 \pm 2.9$ years & $3.7 \pm 3.2$ years & NS \\
\hline Sex & 694 males, 563 females & 226 males, 82 females & \\
\hline$\%$ of females & 44.8 & 26.6 & $p<0.001$ \\
\hline Side of hernia & 745 right, 456 left, 56 bilateral & 177 right, 117 left, 14 bilateral & \\
\hline$\%$ of laterality & Right (58.4), left (35.8), bilateral (4.4) & Right (57.5), left (38), bilateral (4.5) & NS \\
\hline \multicolumn{4}{|c|}{ Associated morbidities necessitate combined operation } \\
\hline Maldescended testis & $41(3.3 \%)$ & $11(3.6)$ & NS \\
\hline Umbilical hernia/cyst & $149(11.9 \%)$ & $9(2.9 \%)$ & $p<0.001$ \\
\hline \multicolumn{4}{|l|}{ Visceral sliding/incarceration } \\
\hline Omentum & $15(1.2 \%)$ & $1(0.3 \%)$ & NS \\
\hline Bowel loop, cecum, appendix & $14(1.1 \%)$ & $2(0.6 \%)$ & NS \\
\hline Ovarium, ovarian duct & $42(3.3 \%)$ & $10(3.2 \%)$ & NS \\
\hline \multicolumn{4}{|l|}{ After primary herniorrhaphy } \\
\hline Recurrence & $7(0.6 \%)$ & $2(0.6 \%)$ & NS \\
\hline Contralateral hernia & $26(2.1 \%)$ & $4(1.3 \%)$ & NS \\
\hline Miscellaneous & $15(1.2 \%)$ & $9(2.9 \%)$ & NS \\
\hline
\end{tabular}

$L P C$ laparoscopic patent processus vaginalis closure, $C D$ cut-down herniorrhaphy, $N S$ not significant

Table 2 Operative findings

\begin{tabular}{|c|c|c|c|c|c|}
\hline & \multirow[t]{2}{*}{ LPC } & \multicolumn{3}{|l|}{$\mathrm{CD}$} & \multirow[t]{2}{*}{ Difference } \\
\hline & & Total & $\mathrm{CDL}$ & CDA & \\
\hline \multicolumn{6}{|l|}{ Laterality of $P P V$} \\
\hline Right & 425 & 143 & 111 & 32 & \\
\hline Left & 211 & 99 & 78 & 21 & \\
\hline Bilateral & 621 & 66 & 57 & 9 & \\
\hline Total & 1878 & 374 & 303 & 71 & \\
\hline$\%$ of contralateral PPV & 47 & & 21.6 & & $p<0.001$ \\
\hline \multicolumn{6}{|c|}{ Operation time (mean $\pm S D$, $\min$ ) } \\
\hline Unilateral & $\begin{array}{l}n=591 \\
28.2 \pm 9.2\end{array}$ & $\begin{array}{l}n=216 \\
27.8 \pm 13.5\end{array}$ & $\begin{array}{l}n=171 \\
29.1 \pm 12.3\end{array}$ & $\begin{array}{l}n=45 \\
23.1 \pm 16.8\end{array}$ & \\
\hline Difference & NS & & $p<0.05$ & & \\
\hline Bilateral & $\begin{array}{l}n=542 \\
35.8 \pm 11.6\end{array}$ & $\begin{array}{l}n=60 \\
46.7 \pm 17.7\end{array}$ & $\begin{array}{l}n=50 \\
48.1 \pm 18.3\end{array}$ & $\begin{array}{l}n=10 \\
39.5 \pm 15\end{array}$ & \\
\hline Difference & $p<0.001$ & & $p<0.05$ & & \\
\hline Male versus female & Male & & Female & & \\
\hline Unilateral LPC & $n=345$ & $30 \pm 8$ & $n=246$ & $25.6 \pm 10$ & $p<0.001$ \\
\hline Bilateral LPC & $n=253$ & $39.5 \pm 10.6$ & $n=289$ & $32.6 \pm 11.5$ & $p<0.001$ \\
\hline Unilateral CD & $n=154$ & $28 \pm 12.1$ & $n=62$ & $27.3 \pm 16.6$ & NS \\
\hline Bilateral CD & $n=43$ & $48.6 \pm 18.5$ & $n=17$ & $41.8 \pm 14.1$ & NS \\
\hline
\end{tabular}

$L P C$ laparoscopic patent processus vaginalis closure, $C D$ cut-down herniorrhaphy, $N S$ not significant

spermatic duct and ovarian duct, which was anastomosed under surgical microscopy during the same session. There was a statistically significant difference in the incidence of intraoperative injuries to the reproductive system between the LPC and CD groups (0\% vs. $0.6 \%$, $p<0.005)$. 


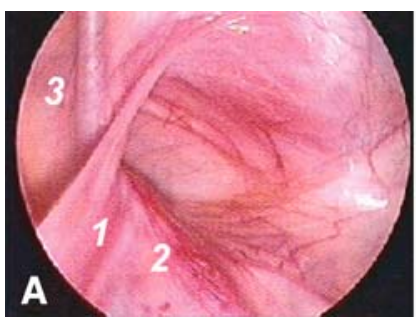

Fig. 4 G.K., 2-year-old boy right indirect inguinal hernia. A Preoperative findings; 1 spermatic cord, 2 testicular vessels, 3 inferior epigastric vessels. B Immediately after closure; 4 Umbilical plica. Umbilical plica has been drawn toward the center of ligation. The spermatic cord and testicular vessels run apart from the ligation.

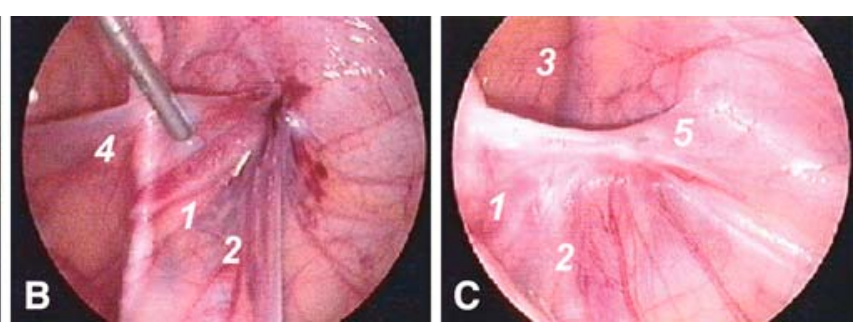

C Revisit for contralateral hernia developed after 3 months; 5 suture knot. The primarily closed IIR has been covered by thick cicatricial tissue resulting in super-high ligation. The spermatic cord and testicular vessels have returned to preoperative places

the floor of the inguinal canal due to operative trauma; (3) failure to close the internal ring in girls; and (4) postoperative wound infection and hematoma [10]. The laparoscopic technique has proven to be a method that can avoid all these possible causes of recurrence [11]. The third problem is injury to the reproductive system. Childhood inguinal herniorrhaphy has been said to be one of the most frequent causes of infertility [12].

The goal of our project was based on the principle of the traditional cut-down technique, which involves completely extraperitoneal high ligation of the PPV, minimizing the above-mentioned drawbacks with a simple technique. We devised the needles to accomplish circumferential ligation of the PPV via the extraperitoneal route more easily, safely, and completely. The needle goes beneath the ligamentum teres uteri distal to the U-turned ovarian duct in girls, involving the ligament inside of the ligature. To avoid damage to the spermatic cord and testicular vessels in boys, the needle is advanced between the peritoneum and cord and vessels.

At the preoperative guidance session, three methods were proposed to the patients' parents: traditional cut-down repair, additional diagnostic laparoscopy for contralateral IIR inspection with simultaneous closure of a cPPV, and laparoscopic repair. The parents chose the laparoscopic repair more frequently based on the reasons described in the Results, which were occasioned by the predominance of girls and associated morbidities of the umbilicus in the LPC group. The parents were very satisfied with the wound cosmesis in LPC group patients. Hand-in-hand with experience-related advances in technique and patient feedback to family doctors, the incidence of LPC being chosen has markedly increased and the procedure garnered the position of the new standard of herniorrhaphy in our hospital.

Regarding the intraoperative findings, incidences of cPPV have been reported to range from 20-40\% [13-16]. The outstanding point in our series was the difference of cPPV rates between the CDL (21.6\%) and LPS (47\%) groups. This difference might be due to the technical 
differences of laparoscopic examination. In diagnostic laparoscopy during the cut-down procedure, an endoscope was inserted through the ipsilateral hernia sac with or without an assistant grasper. On the other hand, during an LPS procedure the laparoscope through the umbilicus is capable of visualizing both IIRs directly and always with the assistance of a grasper. Grossmann et al. described some difficulty in visualization of the contralateral side with an endoscope inserted from the ipsilateral hernia sac [17]. Furthermore, after experiences of contralateral development of clinical hernia in two girls, we have adopted more strict criteria for negative cPPV in LPC.

The average operation time for a unilateral hernia in the LPS group was comparable with the CD group, but was shorter for bilateral hernias. According to the difference in time between unilateral and bilateral closure, the time needed for closure of the IIR itself accounted for $8 \mathrm{~min}$. The time for diagnostic laparoscopy via an ipsilateral hernia sac in cut-down herniorrhaphy was calculated at 6 min, which is compatible with a previous report [13]. Differences in time between boys and girls came from the care required around the spermatic cord. Because of tight contact between the spermatic cord and the peritoneum, separation of these structures in advance using electrocautery and a grasper is advisable, although in skilled hands, this step can be abridged, the result of which saves 4 min for a unilateral repair.

As for postoperative complications, contralateral hernia developed in eight children who underwent LPC or CDL. The patency had been overlooked in two of them because of a peritoneal veil covering the orifice completely. The remaining six children had had a pinhole orifice or shallow depression, development of a metachronous hernia from which was not thought to occur. The incidence of metachronous hernia of children who underwent laparoscopic inspection was $1.1 \%$, which was far less compared with reported traditional unilateral open repair.

It is very difficult to eradicate postoperative recurrence as a fate of herniorrhaphy. Despite the fact that the laparoscopic approach theoretically provides high ligation of the PPV more proximally than open repair, higher rates of recurrence have been reported with this approach. Laparoscopic repairs involving closure of the hernia opening by suturing within the abdominal cavity in the pursestring or Z-type suture fashion where the suture material is tied off intracorporeally may have an intrinsic risk of reopening the vaginal process, leading to the recurrence of the hernia or development of a hydrocele. Reported recurrence rates were 3.1-4.4\% [18-20]. In another procedure in which a circuit suture was placed extraperitoneally around the hernial orifice, crossing over the spermatic cord or the testicular vessels to leave them untouched and spare them from injury, small spaces are left above these structures.
Reported recurrences in these techniques are $0.8-2.8 \%$, which is lower than intraperitoneal closures [21-23]. Methods that allow complete encircling of the PPV, such as the intraperitoneal pursestring stitch passing between the peritoneum and the cord and vessel structures so as not to leave any skipped area, or a laparoscopic technique that produces every step of the open procedure involving complete division and stitching up of the PPV at the IIR, achieved the lowest recurrence rate from $0-1.3 \%$ $[11,24]$.

Our technique has fundamentally the same concept as the latter-mentioned techniques, but with more simplicity. Hernia recurrence occurred in two boys in the LPC group $(0.2 \%)$ : one due to early rejection of the suture, and the other due to provable loosening of the suture knot, prompting a modification in technique. The modification was made by double-ligation of the proximal end of the sac for infants younger than 1 year 6 months in whom the external inguinal ring is located so close to the IIR that the shortened inguinal canal becomes uncovered by musculature and is vulnerable to increased intra-abdominal pressure. The doubly ligated IIR is expected to hold against pressure until wound healing is completely accomplished.

Drawbacks associated with the reproductive system are a hidden but not negligible problem. One report suggests that vas deferens or epididymis was found in $0.53 \%$ of hernial sacs removed during herniorrhaphy [25]. The incidence of vasal injury during inguinal herniorrhaphy has been estimated at $0.5 \%$ [26]. Fallopian tube obstruction in a woman with a history of childhood bilateral inguinal herniorrhaphy was reported as the cause of infertility [27]. We had two episodes of injury to the reproductive system during cut-down repair. In addition, testicular atrophy, ascent of the testis, ovarian malposition, and bladder injury have been reported, none of which occurred in our laparoscopic series.

Despite increasing reports regarding the laparoscopic approach, there has been only one comparative study between laparoscopic and open repair. Chan et al. emphasized the superiority of laparoscopic repair from the points of less pain, prompter recovery, and better cosmesis [28]. Operation times were longer for unilateral and equal for bilateral repair compared with open repair. In our series, the majority of parents preferred laparoscopic repair based on postoperative cosmetic superiority, bilateral IIR inspection, and simultaneous repair for an unpleasantlooking umbilicus. Laparoscopic operation times were equal for unilateral and shorter for bilateral repair compared with open repair. There was no injury to the reproductive system in contrast to the cut-down procedure. Postoperative recurrence and contralateral hernia were less in the laparoscopic group, although we could not achieve $0 \%$ incidence. 


\section{Conclusions}

Although we must currently accept that laparoscopic hernia repair has not been around long enough to rate fully the risk of late complications, we believe that this procedure with the Endoneedle can be a routine procedure with results comparable or superior to those with open procedures. The advantages of our technique include the following: technically simple, short operation time, inspection of bilateral IIRs with simultaneous closure of CPPV, the reproductive system remains intact, routine addition of umbilicoplasty if desired by the parents of patients, and essentially indiscernible wounds.

Open Access This article is distributed under the terms of the Creative Commons Attribution Noncommercial License which permits any noncommercial use, distribution, and reproduction in any medium, provided the original author(s) and source are credited.

\section{References}

1. Potts WJ, Riker WL, Lewis JE (1950) The treatment of inguinal hernia in infants and children. Ann Surg 132:566-576

2. Skinney MA, Grosfeld JL (1993) Inguinal and umbilical hernia repair in infants and children. Surg Clin North Am 73:439-449

3. Ingimarsson O, Spak I (1983) Inguinal and femoral hernias: longterm results in a community hospital. Acta Chir Scand 149:291297

4. Rowe MI, Clatworthy HW (1971) The other side of the pediatric inguinal hernia. Surg Clin North Am 51:1371-1376

5. Burd RS, Heffington SH, Teague JL (2001) The optimal approach for management of metachronous hernias in children: a decision analysis. J Pediatr Surg 36:1190-1995

6. Given JP, Rubin SZ (1989) Occurrence of contralateral inguinal hernia following unilateral repair in a pediatric hospital. J Pediatr Surg 24:963-965

7. Endo M, Ukiyama E (2001) Laparoscopic closure of patent processus vaginalis in girls with inguinal hernia using a specially devised suture needle. Pediatr Endosurg Innov Tech 5:187-191

8. Rowe MI, Lloyd DA (1986) Inguinal hernia. In: Welch KJ, Randolph JG, Ravitch MM, O'Neil JA Jr, Rowe MI (eds) Pediatric surgery, 4th edn. Year Book Medical Publishers, Chicago, London, pp 779-793

9. Surana R, Puri P (1993) Is contralateral exploration necessary in infants with inguinal hernia? J Pediatr Surg 28:1026-1027

10. Grosfeld JL, Minnick K, Shedd F, West KW, Rescoria FJ, Vane DW (1991) Inguinal hernia in children: factors affecting recurrence in 62 cases. J Pediatr Surg 26:283-287

11. Chan KL, Tam PKH (2004) Technical refinements in laparoscopic repair of childhood inguinal hernia. Surg Endosc 18:957960
12. Matsuda T (2000) Diagnosis and treatment of post-herniorrhaphy vas deferens obstruction. Int J Urol 7(Suppl):S35-S38

13. Miltenburg DM, Nuchtern JG, Jaksic T, Kozinetiz C, Brandt ML (1998) Laparoscopic evaluation of the pediatric inguinal hernia: a meta-analysis. J Pediatr Surg 33:874-879

14. Holcomb GW III, Brock JW III, Morgan WM III (1994) Laparoscopic evaluation for a contralateral patent processus vaginalis. J Pediatr Surg 29:970-974

15. Lotan G, Efrati Y, Stlero S, Klin B (2004) Transinguinal laparoscopic examination: an end to the controversy on repair of inguinal hernia in children. IMAJ 6:339-341

16. Sozubir S, Ekingen G, Senel V, Kohraman H, Guvenc BH (2006) A continuous debate on contralateral processus vaginalis: evaluation technique and approach to patency. Hernia 10:74-78

17. Grossmann PA, Wolf SA, Hopkins JW, Paradise NP (1995) The efficacy of laparoscopic examination of the internal inguinal ring in children. J Pediatr Surg 30:214-218

18. Chinnaswamy P, Malladi V, Jani KV, Parthasarthi R, Shetty RA, Kavalakat AJ, Prakash A (2005) Laparoscopic inguinal hernia repair in children. JSLS 9:393-398

19. Schier F (2006) Laparoscopic inguinal hernia repair: a prospective personal series of 542 children. J Pediatr Surg 41:1081-1084

20. Schier F, Montupet P, Esposito C (2002) Laparoscopic inguinal herniorrhaphy in children. A three-center experience with 933 repairs. J Pediatr Surg 37:395-397

21. Patkowski D, Czernik J, Chrzan R, Jaworski W, Apoznanski W (2006) Percutaneous internal ring suturing: a simple minimally invasive technique for inguinal hernia repair in children. $\mathrm{J}$ Laparoendosc Adv Surg Tech 16:513-517

22. Spurbeck WW, Prasad R, Lobe TE (2005) Two-year experience with minimally invasive herniorrhaphy in children. Surg Endosc 19:551-553

23. Takehara H, Yakabe S, Kameoka K (2006) Laparoscopic percutaneous extraperitoneal closure for inguinal hernia in children: clinical outcome of 972 repairs done in 3 pediatric surgical institutions. J Pediatr Surg 41:1999-2003

24. Becmeur F, Philippe P, Lemandat-Schultz A, Moog R, Grandadam S, Lieber A, Toledano D (2004) A continuous series of 96 laparoscopic inguinal hernia repairs in children by a new technique. Surg Endosc 18:1738-1741

25. Steigman CK, Sotelo-Avila C, Weber TR (1999) The incidence of spermatic cord structures in inguinal hernia sacs from male children. Am J Surg Pathol 23:880-885

26. Pasqualotto FF, Pasqualotto EB, Agarwal A, Thomas AJ Jr (2003) Results of microsurgical anastomosis in men with seminal tract obstruction and infertility. Rev Hosp Clin Fac Med Sao Paulo 58:305-309

27. Hansen KA, Eyster KM (2006) Infertility: an unusual complication of inguinal herniorrhaphy. Fertil Steril 86:217-218

28. Chan KL, Hui WC, Tam PKH (2005) Prospective, randomized, single-center, single-blind comparison of laparoscopic vs. open repair of pediatric inguinal hernia. Surg Endosc 19:927-932 